\title{
Autism-linked genetic variants increase, decrease intelligence
}

\author{
BY LAURA DATTARO
}

19 OCTOBER 2021

\section{Listen to this story:}

https://www.spectrumnews.org/wp-

content/uploads/2021/10/audio-95497b03-d33d-433c-86d b-af06eae4a337-encodings.mp3

Common and rare variants in or near certain autism-associated genes can have opposite effects on cognition. Likewise, some autistic people carry autism-linked variants with opposite effects on a measure of intelligence.

Two independent teams presented the unpublished findings yesterday at the 2021 American Society of Human Genetics conference, which is taking place virtually for the second year in a row.

A mix of common and rare variants work together to shape a person's chances of having autism, previous research shows. Common variants, which exert subtle effects on a person's traits, tend to be associated with a high intelligence quotient (IQ). Rare variants, by contrast, have stronger effects and typically track with a below-average IQ. But it has been unclear how variants that contribute to the same condition could have such different effects on cognition.

Teasing apart that conundrum could help researchers better understand the genetic underpinnings of the wide variation of traits seen in autistic people, says Daniel Weiner, a doctoral candidate in Elise Robinson's lab at the Broad Institute in Boston, Massachusetts, who presented some of the work.

"This sort of approach is one way of starting to think about how there may be convergence of common and rare variation in autism," Weiner says.

\section{Opposite effects:}




\section{Spectrum | Autism Research News}

https://www.spectrumnews.org

Weiner and his colleagues analyzed the whole-genome sequences of 5,048 people with autism and their parents, all of whom participate in the Simons Simplex Collection (SSC) and Simons Powering Autism Research for Knowledge (SPARK). Both projects are funded by the Simons Foundation, Spectrum's parent organization. The researchers also separately analyzed the sequences of 4,334 autistic people and their parents from the Psychiatric Genomics Consortium (PGC), a study of 11 neurodevelopmental and psychiatric conditions, including autism, based at the University of North Carolina at Chapel Hill.

They looked for common variants associated with high educational attainment — a proxy for high IQ — in and near 255 genes associated with autism; rare, loss-of-function mutations in these genes are known to be associated with low IQ. They used a statistical method they created several years ago, called the polygenic transmission disequilibrium test, to determine the extent to which the autistic children had inherited these common variants.

On average, autistic children inherit more common, IQ-boosting variants in or near autism-linked genes than would be expected by chance, the analysis shows, and that pattern holds in both the Simons and PGC sets of sequences.

"Even at these genes where disruption of them is associated with decreased IQ in [autism], we are still seeing over-transmission, on average, of IQ-increasing common variant risk," Weiner says.

\section{Separate pathways:}

In a separate analysis, another team compared the sequences of 6,929 autistic people and 32,663 non-autistic people with similar IQs; the data came from the SSC and SPARK cohorts, plus four others.

Autistic people were more likely than their non-autistic peers to have large, rare mutations that decrease IQ, the team found. They were also more likely to have common variants that increase $I Q$.

Adjusting for differences in the number of intelligence-associated common variants did not shift the large mutations' effects on an individual's chances of having autism, the study shows.

"Importantly, these results suggest that these two genetic factors may confer autism risk independently," said Zoe Schmilovich, a doctoral candidate in Guy Rouleau's lab at McGill University in Montreal, Canada, when presenting the work.

The two analyses together suggest that distinct biological pathways underpinning differences in cognition may be contributing to autism, Weiner says.

Read more reports from the 2021 American Society of Human Genetics annual meeting. 


\section{Spectrum | Autism Research News}

https://www.spectrumnews.org

Cite this article: https://doi.org/10.53053/WCXO7531 